\title{
Automated System for Purification of Dye- Terminator Sequencing Products Eliminates Up-Stream Purification of Templates
}

BioTechniques 26:980-983 (May 1999)

\author{
Bjørn Magne Fangan'1, Ole Jesper Dahlberg², Arne Helge Deggerdal, Marie Bosnes \\ and Frank Larsen ${ }^{2}$ \\ Dynal A.S., Oslo, ${ }^{1}$ FluiGene, Skreia and ${ }^{2}$ GenoVision Cytomics A.S., Oslo, Norway
}

\begin{abstract}
The quality of sequencing results is to a large extent determined by the purity of the template and the purification of the sequencing products. Fragments that can act as unspecific primers and templates are removed before gel analysis, and the background of unspecific signals is highly reduced. Purification of the sequencing products is needed to remove salts, nucleotides, proteins and template DNA that can interfere with the gel separation. We have developed a product, DYNAPURE ${ }^{\mathrm{TM}}$ Dye Terminator Removal, that specifically isolates and purifies the sequencing products in $10 \mathrm{~min}$. The method is based on biotinylated sequencing primers and superparamagnetic streptavidin beads. A PCR product is sequenced using a biotinylated sequencing primer, and the sequencing products are then bound to streptavidin beads in a 5-min reaction. The bead-DNA complexes are magnetically separated from the rest of the solution, and the remaining buffer constituents are washed away with TE buffer or with $70 \%$ ethanol. The whole procedure can be automated on liquid-handling robots fitted with a magnet station. The method eliminates purification of templates before cycle sequencing.
\end{abstract}

\section{INTRODUCTION}

The most convenient way to do automated DNA sequencing today is by the dye terminator chemistry. Every reaction is done in one tube, any primer can be used and fundamental improvements have been made to the sequencing enzymes to make them incorporate dye terminators more efficiently. The most important factors in getting successful sequencing results with a low unspecific background, however, are still the quality of the template and effective removal of excess dye terminators and salts before gel loading $(2,3)$. Primers that are left over from the polymerase chain reaction (PCR) amplifica- tion will also prime DNA polymerization, and any molecule able to do so nonspecifically adds to the background; all the terminated fragments, specific and nonspecific, carry a fluorescent label. This can lead to poor sequencing ladders with a high background of nonspecific sequencing products. Direct cycle sequencing of PCR products is therefore dependent on well-optimized PCRs to be successful. One way to circumvent these problems is to purify the PCR products before sequencing. PCR product purification is mostly done with some kind of spin column, by ethanol-precipitation or by enzymatic removal of single-stranded DNA fragments (mostly primers). Before separation of the labeled fragments on a sequencing gel or a capillary electrophoresis matrix, salts and nucleotides have to be removed from the extension products. This step is today most often done with ethanol-precipitation or spin column purification.

Here, we demonstrate that by introducing a biotinylated sequencing primer and capturing the resulting biotinylated sequencing products onto streptavidin-coated Dynabeads ${ }^{\circledR}$, both upstream purification of sequencing templates and downstream ethanol-precipitation of sequencing products can be eliminated. The procedure is completed in less than $10 \mathrm{~min}$ and is well suited for automation on several robotic workstations. The method is highly specific, and results show that even suboptimal PCRs can be used for sequencing successfully.

\section{MATERIALS AND METHODS}

Identical samples were subjected to dye-terminator cycle sequencing with biotinylated sequencing primers and either purified using streptavidin-coated Dynabeads or ethanol-precipitation of the sequencing products. The two purification 
systems were tested on two templates of different purity: $(i)$ purified and non-purified PCR products of a 1-kb human DNA cloned in pUC18 and (ii) non-purified PCR products after amplification of exon 13 of the cystic fibrosis transmembrane conductance regulator (CFTR) gene.

\section{PCR Amplifications}

Plasmid amplifications were carried out using 5 pmol of PCR primers complementary to the conserved sequences upstream and downstream of the multiple cloning site in pUC18, forward: 5'-CGC CAG GGT TTT CCC AGT CAC GAC G-3'; reverse: 5'-GCT TCC GGC TCG TAT GTT GTG TGG- $3^{\prime}$. The reactions were run for 25 cycles at $95^{\circ}, 65^{\circ}$ and $72^{\circ} \mathrm{C}$ for 30,30 and $120 \mathrm{~s}$, respectively. Genomic PCRs of the CFTR gene were run for 34 cycles at $94^{\circ}, 55^{\circ}$ and $72^{\circ} \mathrm{C}$ for 30,15 and $60 \mathrm{~s}$, respectively, using 5 pmol of previously published primers (1). The PCR products were analyzed before sequencing on $3 \%$ NuSieve ${ }^{\circledR}$ 3:1 agarose gels (FMC BioProducts, Rockland, ME, USA).

\section{Purification of PCR Products}

Purification of PCR product for sequencing was performed using Amicon ${ }^{\circledR}$ Centricon ${ }^{\circledR} 100$ columns (Millipore, Bedford, MA, USA).

\section{Dye-Terminator Cycle Sequencing}

Eight microliters of purified and non-purified PCR products of cloned human DNA were sequenced using 3.2 pmol of a biotinylated universal forward sequencing primer: $5^{\prime}$-GTT TTC CCA GTC ACG AC-3'. Biotinylated nested primer (3.2 pmol) was used (5'-Biotin-GGT CAC TTC TAA AAT GGA AC-3') for direct sequencing of $8 \mu \mathrm{L}$ crude CFTR PCR product. This primer was designed using the OLIGO ${ }^{\mathrm{TM}}$ Version 4.05 (National Biosciences, Plymouth, MN, USA). Standard dye-terminator cycle sequencing reactions were run directly on non-purified PCR products using the ABI PRISM ${ }^{\mathrm{TM}}$ Dye Terminator Cycle Sequencing Kit with AmpliTaq ${ }^{\circledR}$ DNA Polymerase, FS following the manufacturer's recommendations (both from PE Biosystems, Foster City, CA, USA).

\section{Purification of Sequencing Products}

Magnetic-bead purification of sequencing products was performed by using the DYNAPURETM Dye Terminator Removal Kit (Dynal A.S., Oslo, Norway) as follows: $50 \mu \mathrm{g}(5$ $\mu \mathrm{L}$ ) of Dynabeads M-280 Streptavidin ${ }^{\mathrm{TM}}$ (Dynal A.S.) were added to $15 \mu \mathrm{L}$ high-salt, binding buffer $(10 \mathrm{mM}$ Tris- $\mathrm{HCl}$, $\mathrm{pH}$ 7.5, 1.0 mM EDTA, 2.0 M NaCl). This bead suspension $(20 \mu \mathrm{L})$ was then added to the completed sequencing reactions and incubated for $5 \mathrm{~min}$ at room temperature for the biotinylated sequencing products to bind to the streptavidin beads. The magnetic particles were subsequently collected from the supernatant using a magnetic particle concentrator (Dynal MPC ${ }^{\circledR}$; Dynal A.S.). The DNA-bead complexes were washed once in $50 \mu \mathrm{L}$ TE buffer (or 70\% ethanol if using BigDye $^{\mathrm{TM}}$ chemistry; PE Biosystems) by pipetting up and down five times and then resuspended in $5 \mu \mathrm{L}$ loading buffer (formamide: $50 \mathrm{mM}$ EDTA, $\mathrm{pH}$ 8.0, 5:1). Biotinylated sequencing products were eluted off the beads by heating at $80^{\circ} \mathrm{C}$ for $2 \mathrm{~min}$, and the whole mixture was loaded on to the sequencing gel. As a control, sequencing products were purified by ethanol-precipitation according to PE Biosystems' recommendations. Remaining ethanol was then evaporated in SpeedVac ${ }^{\circledR}$ (Savant Instruments, Holbrook, NY, USA). Purified sequencing products were resuspended in $5 \mu \mathrm{L}$ loading buffer, denatured and analyzed on $6 \%$ polyacrylamide gels on Model 373A DNA Analysis System (PE Biosystems).

\section{Automation}

Magnetic-bead purification was automated on a Biomek ${ }^{\circledR}$ 2000 Laboratory Automation Workstation (Beckman Coulter, Fullerton, CA, USA), using an MP200 pipetting tool. Purification was performed in polyvinylcarbonate (PVC) u-bottom 96-well plates (Falcon ${ }^{\circledR}$ Model 3911; Becton Dickinson Labware, Bedford, MA, USA). Volumes and incubation times were as described above for the manual system. The protocol was written in Bioworks Version 1.4, except for two resuspension steps, which were written in Bioscript (both from Beckman Coulter).

To take advantage of the magnetic properties of the Dynabeads, we used a magnet station (Dynal MPC-auto96; Dynal A.S.) consisting of a rack for microplates and a lift shifting the magnets between an upper and a lower position. In the upper position, the magnets come up between the wells of microplates, and all the particles in the wells are attracted to the magnets. In the lower position, the distance between the wells and the magnets is too large for any such interaction to take place. The device is operated by a small electrical motor that can be controlled through one of the communication ports of the Biomek Workstation. Raising and lowering the magnets is done by simple SEND TO DEVICE statements in Bioworks. The magnet station fits in any of the Biomek's labware holders.

\section{Data Analysis}

Collected data were analyzed using the ABI 373A Analysis Software Version 1.2.1 (PE Biosystems). Analyzed sequences have been left unedited.

\section{RESULTS AND DISCUSSION}

To avoid tedious and costly template preparation and purification of sequencing products, we have developed the DYNAPURE Dye Terminator Removal Kit, which allows direct dye-terminator cycle sequencing on non-purified PCR products and eliminates ethanol-precipitation of sequencing products. This is achieved by sequencing the template with a biotinylated primer and capturing the sequencing products with streptavidin-coated Dynabeads (2). Since a biotinylated sequencing primer is used, the sequencing products can be specifically separated from a mixture of contaminating nonbiotinylated sequencing products. To test the performance of the purification system, we have run sequencing reactions on both purified and non-purified PCR templates.

The results of dye-terminator sequencing of purified and non-purified PCR products from cloned human DNA are presented in Table 1 . The purification system using magnetic beads is compared to ethanol-precipitation of sequencing products. These results show that when ethanol precipitations are used for purification of sequencing products, improvements in read length and accuracy are achieved when the PCR products 
Table 1. Comparison of DYNAPURE and Ethanol Precipitation for Purification of Sequencing Products

\begin{tabular}{|c|c|c|c|c|c|}
\hline & $\begin{array}{l}\text { Number of } \\
\text { Sequences }\end{array}$ & $\begin{array}{l}\text { Average Read } \\
\text { Length }\end{array}$ & SDa & $\begin{array}{l}\text { Average Number } \\
\text { of Ambiguities }\end{array}$ & SDa \\
\hline $\begin{array}{l}\text { Ethanol precipitation, } \\
\text { purified template }\end{array}$ & 18 & 499.1 & 4.59 & 4.05 & 2.20 \\
\hline $\begin{array}{l}\text { Ethanol precipitation, } \\
\text { unpurified template }\end{array}$ & 18 & 472 & 9.69 & 15.05 & 2.60 \\
\hline $\begin{array}{l}\text { DYNAPURE, } \\
\text { purified template }\end{array}$ & 24 & 502.5 & 4.58 & 3.92 & 1.98 \\
\hline $\begin{array}{l}\text { DYNAPURE, } \\
\text { unpurified template }\end{array}$ & 24 & 500.5 & 3.06 & 4.46 & 2.96 \\
\hline astandard deviation. & & & & & \\
\hline
\end{tabular}

are purified. The improvement in read length is on average 17 bases, and the reduction of ambiguities is on average 11. Approximately the same read length and accuracy for both purified and non-purified PCR products were seen when utilizing the magnetic purification procedure. This is mostly due to a reduced background of nonspecific sequencing products, but it also reflects an elimination of co-precipitation of dye terminators, which is often seen with ethanol precipitation.

It has been pointed out earlier $(2,3)$ that salt, residual template and dye terminators will affect the gel analysis of the final sequence. The effect is not as pronounced with a cloned human template, as with more complex and difficult templates such as genomic PCR products. To fully test the advantages of the method, genomic PCRs were run on exon 13 of the CFTR gene. Figure 1 shows the results from direct sequencing of these amplification products with and without streptavidin-bead purification of the sequencing products. The two samples were treated identically, except that sample A was precipitated directly, whereas sample B was purified with magnetic beads. As can be seen from the sequence, the bead-based method eliminates the need for other purification procedures.

Our results show that direct cycle sequencing of non-purified, genomic PCR products can give sequences that cannot be interpreted (Figure 1A), although the PCR products appear specific on an agarose gel. If the biotinylated sequencing products are specifically isolated from the rest of the sequencing reaction with DYNAPURE Dye Terminator Removal a clear, fully interpretable sequence is obtained (Figure 1B).

Good sequencing results have traditionally depended on two purification steps: (i) an upstream purification of the template to be sequenced to reduce the amount of unspecific sequencing products; (ii) a downstream purification of the sequencing products to remove salts and unincorporated dye terminators that interfere with the gel separation. The purification systems have imposed high cost and time-consuming procedures on the users. We have developed a system for purification of sequencing products that also eliminate the need

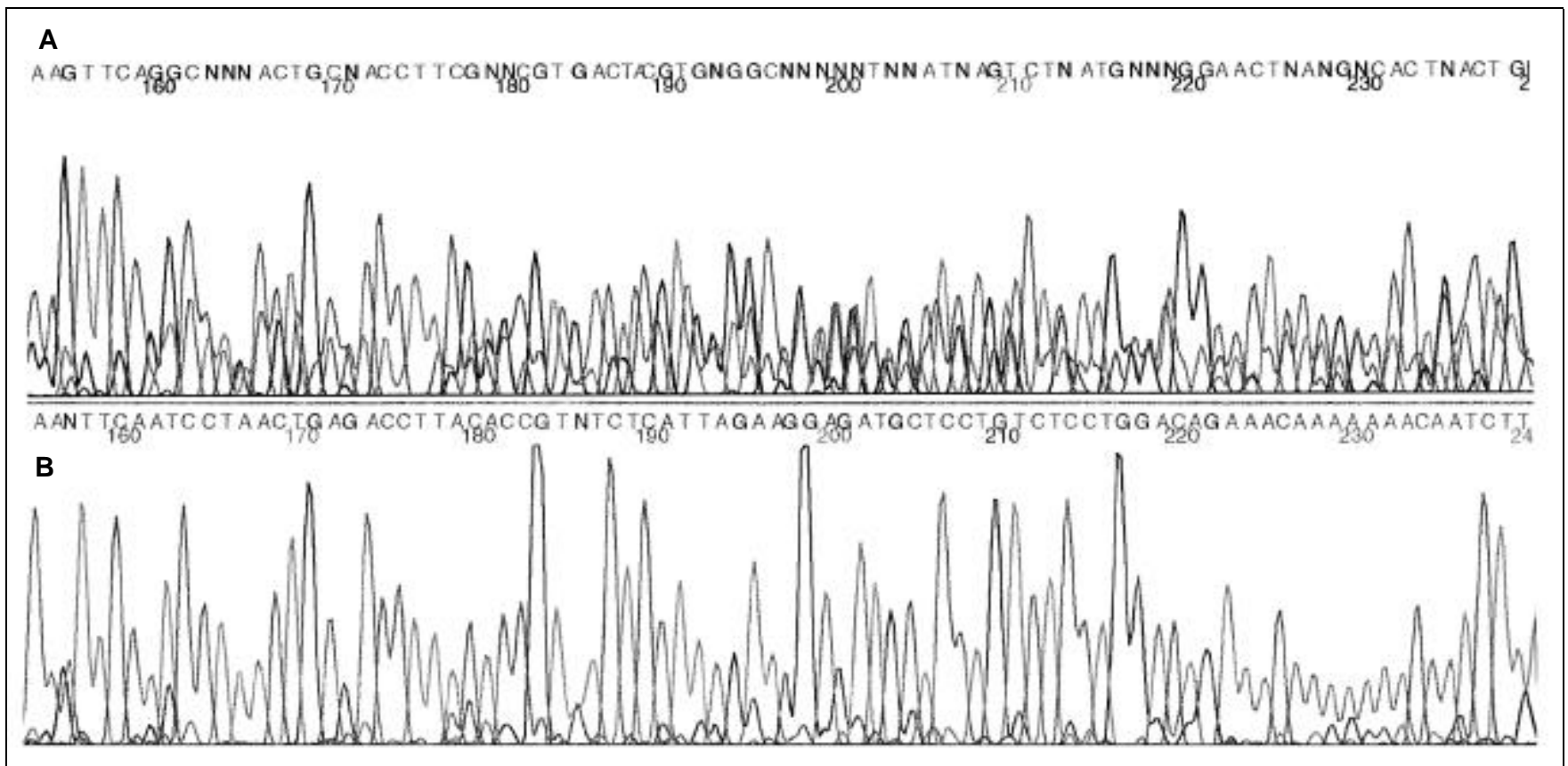

Figure 1. Purification of dye-terminator sequencing products from non-purified PCR amplifications of exon 13 of the CFTR gene. (A) Upper panel: standard dye-terminator sequencing with ethanol-precipitation of the sequencing products. (B) Lower panel: same sample purified with DYNAPURE Dye Terminator Removal. 
for upstream purification of templates for sequencing. By biotinylating the sequencing primer, only the biotinylated sequencing products are captured onto the streptavidin-coated Dynabeads. When the sequencing products are bound to the beads, they are easily manipulated, and all traces of unincorporated terminators, non-biotinylated sequencing products and other contaminating substances are readily washed away. The less than 10-min purification method is performed in one tube without any centrifugation step. Since PCR products can be sequenced directly, DYNAPURE Dye Terminator Removal gives a great advantage in sequence-gap-filling procedures. In capillary electrophoresis where the electric charge of the DNA molecules is utilized in loading the sample, it is extremely important to remove all salts from the solution. For this purpose, solid-phase purification is most suitable since it effectively washes the sequence products while attached to magnetic beads. This will, however, require that the beadDNA complexes are washed in 70\% ethanol and are resuspended in a salt-free loading solution.

With the increasing number of high-throughput sequencing labs, the need for automation of the purification system is obvious. We have developed a protocol for dye-terminator cycle sequencing reaction purification that can be run in a 96well format on a Biomek 2000 robot fitted with a Dynal MPC-auto96 Magnet Station. The magnet station can be fitted on all liquid-handling robots with labware holders that can take standard microplates. It can be controlled from any software that can directly access a serial port. The method will be easy to implement on multichannel liquid-handling workstations, which means that multiple processing of samples in a 96-, 384- or even 1536-well format can be completed in less than $10 \mathrm{~min}$. The same tips can be used throughout the procedure, and the reaction is performed in one tube, which reduces the costs when performed on a robotic workstation. Results from these experiments are comparable to the results from manual clean-up (results not shown). The DYNAPURE Dye Terminator Removal protocol therefore presents a solution to make DNA sequencing completely automated.

\section{ACKNOWLEDGMENTS}

We wish to thank Nicola Buckley and Tone Ingvaldsen for useful comments on the manuscript and Dr. Erik Hornes for helpful discussions. Mette Kroken is acknowledged for supplying DNA samples in the early stages of the project.

\section{REFERENCES}

1.Gyllensten, U.B., M. Allen and A. Josefson. 1992. Overview: sequencing of in vitro amplified DNA, p. 1-15. In J. Ellingboe and U.B. Gyllensten (Eds.), The PCR Technique: DNA Sequencing, Eaton Publishing. Natick, Massachusetts.

2.Tong, X. and L.M. Smith. 1992. Solid-phase method for the purification of DNA sequencing reaction. Anal. Chem. 64:2672-2677.

3.Tong, X. and L.M. Smith. 1993. Solid-phase purification in automated DNA sequencing. J. DNA Sequenc. Map. 4:151-162.

Address correspondence to Marie Bosnes, Dynal A.S., P.O. Box 158 Skøyen, 0212 Oslo, Norway. Internet: marie. bosnes@dynal.no 